\title{
Robotic Fault Detection Using Nonlinear Analytical Redundancy
}

\author{
Martin L. Leuschen \\ ECE Dept., Rice Univ. \\ Houston, TX \\ martinl@rice.edu
}

\author{
Joseph R. Cavallaro \\ ECE Dept., Rice Univ. \\ Houston, TX \\ cavallar@rice.edu
}

\author{
Ian D. Walker \\ ECE Dept.,Clemson Univ. \\ Clemson, $\mathrm{SC}$ \\ ianw@ces.clemson.edu
}

\begin{abstract}
In this paper we discuss the application of our recently developed nonlinear analytical redundancy (NLAR) fault detection technique to a two-degree of freedom robot manipulator. NLAR extends the traditional linear AR technique to derive the maximum possible number of fault detection tests into the continuous nonlinear domain. The ability to handle nonlinear systems vastly expands the accuracy and viable applications of the AR technique. The effectiveness of the approach is demonstrated through an example.
\end{abstract}

\section{INTRODUCTION}

Fault detection is important in many robotic applications. Failures of powerful robots, fast robots, or robots in hazardous environments are quite capable of causing significant and possibly irreparable havoc if they are not detected promptly and appropriate action taken. As robots are commonly used because power, speed, or resistances to environmental factors need to exceed human levels, fault detection is a common and serious concern in the robotics arena [2,5-7,9].

This work focuses on a nonlinear version of an observability-based fault detection method known as analytical redundancy (AR). This residual generation method and its nonlinear analog, which we have recently derived [5-7], are attractive because they are formally guaranteed to derive the maximum possible number of independent tests of the consistency of sensor data and past control inputs with the system model. Every observable deviation from the model will be tested for, and every test will contain some elements not observed by other tests.

The standard and often used AR fault detection technique [1] is effectively limited to linear systems. As AR is a model-based technique, it is extremely sensitive to differences between the nominal model behavior and the actual system behavior. A system model with strong nonlinear characteristics, such as a multi-joint robot manipulator, suffers considerably from linearization. This makes effective implementation of the AR technique difficult, as modeling errors will generate significant false error signals when linear AR is applied.

To solve this problem we have used nonlinear control theory [4] to extend the AR principle into the nonlinear realm. Although others have combined AR with nonlinear systems $[8,10]$, they have either considered quite general nonlinear systems, and thus structure present in applications such as robotics is not exploited in the analysis [8] or neglected to preserve the formal guarantees of span and independence discussed above [10]. Our NLAR technique is applicable to differentiable nonlinear systems and preserves the formal guarantees. It also generates considerable improvement in performance over linear $\mathrm{AR}$ when performing fault detection on nonlinear systems. In the following sections, we briefly describe the new NLAR technique, and demonstrate its potential in robotic applications via a simple but representative example.

\section{NONLINEAR ANALYTICAL REDUNDANCY}

Let us begin by defining a nonlinear state-space system with $n$ states, $q$ inputs, and $m$ outputs,

$$
\begin{aligned}
& \underline{\dot{x}}(t)=\underline{f}(\underline{x}(t))+\sum_{i} \underline{g}_{i}(\underline{x}(t)) \cdot \underline{u}(t) \\
& \underline{y}(t)=\underline{h}(\underline{x}(t)) .
\end{aligned}
$$

The corresponding linear system model is

$$
\begin{aligned}
& \underline{\dot{x}}(t)=A \underline{x}(t)+B \underline{u}(t) \\
& \underline{y}(t)=C \underline{x}(t) .
\end{aligned}
$$

We adopt the following model of a robotic manipulator with joint variable vector $\underline{q}$, disturbance $\underline{\zeta}$ and input torque vector $\underline{\tau}$

$$
M(\underline{q}) \underline{\ddot{q}}+N(\underline{q}, \underline{\dot{q}})+\underline{\zeta}=\underline{\tau} .
$$


The core concept of NLAR revolves around $\Omega$, the left null-matrix of the observability

$$
[\Omega(\underline{f}, \underline{g})][\vartheta(\underline{f}, \underline{g})]=\underline{0} \text {. }
$$

where the model-based observability $\vartheta(\underline{f}, \underline{g})$ is calculated using standard linear or nonlinear methods using the model equations. In the linear case, the observation space is generated from the rows of $\left[C, C A, C A^{2} \ldots\right]$ in the standard way [1]. For nonlinear systems, the Lie derivative operation $L_{f} h_{i}$ is used to combine the vector functions $\underline{f}, \underline{g}_{i}$ with elements of $\underline{h}$, and the observation space is generated from appropriate combinations of $L_{k} h_{i}, k \in\left\{\underline{f}, \underline{g}_{1}, \underline{g}_{2}, \ldots\right\}$. For more details, see $[4,7]$.

However, if the system is observable it is also possible to express the observability in terms of sensor readings $\underline{y}$ and control inputs $\underline{u}[1,7]$ in addition to the state and model. This observability will be referred to as $\vartheta(\underline{y}, \underline{u})$. The important aspect of this formulation is the explicit dependence of every element of it on the input-output behavior of the system as it is functioning at the time the observability is calculated. By taking the product of $\vartheta(y, \underline{u})$ with the left null-matrix from equation (4) we can generate the NLAR residual tests

$$
[\Omega(\underline{f}, \underline{g})][\vartheta(\underline{y}, \underline{u})]=\underline{R} .
$$

As $\Omega(f, g)$ is derived from the system equations, while the input-output observability matrix depends on the recent sensor readings and inputs, if the system is observable the result of the above matrix multiplication can be expressed as a set of equations dependent on the known quantities of $\underline{y}$ and $\underline{u}$. If the system is behaving in accordance with the nominal model $\vartheta(\underline{y}, \underline{u})$ will be as similar to $\vartheta(f, g)$ as measurement error and noise allow, and equation (5) will generate near-zero values [1,7]. However, if the system model has become inaccurate due to a fault changing the characteristics of the system, nonzero values will be generated, allowing the fault to be detected.

In fact, since the observability space by definition spans all that can be observed about the system using the current model, it can be shown that NLAR is guaranteed to react to any observable discrepancy $[1,7]$. The basis vectors of the observability space generated from system data $(\vartheta(y, \underline{u}))$ span a space dependent on the current behavior of the system that will span the observability space if the model is correct. The null-space of the model derived observability space $\Omega(\underline{f}, \underline{g})$ spans the space of information you shouldn't be able to see if the system is performing according to the model. Project the basis onto the null space and one instantly reduces a complex stream of sensor and input data into residual signals that show all the deviations from the expected model, and only those deviations.

A conceptual geometric representation of NLAR is shown in fig. 1. For clarity, vectors are used to represent the nonlinear spaces derived from the observability space $\vartheta(\underline{f}, \underline{g})$.

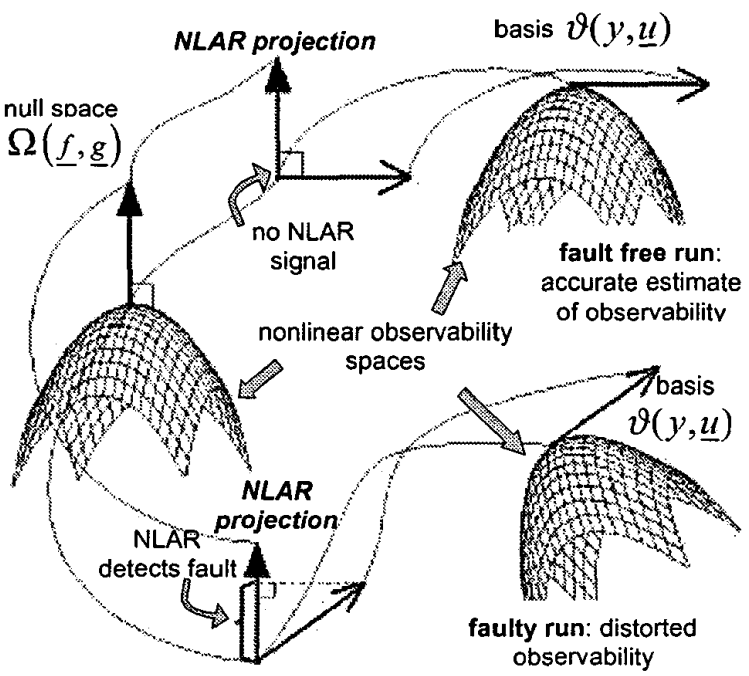

Figure 1: Geometric interpretation of NLAR

NLAR test results can only be zero if the system equations are modeling the system behavior correctly. Any discrepancies, such as those that result from sensor noise, manifest as bias or noise in the NLAR output. However, given a good system model, most faults will cause deviations between the system and the model much greater than the difference caused by modeling inaccuracies. Faults will appear as large nonzero NLAR signals, thus allowing NLAR to be used as an effective tool for fault detection [1,5-7].

The standard linear AR technique described in [1] is similar, but it requires a linear system model like that described in equation (2). This can cause significant extra bias and noise in the linear AR tests if the system has nonlinear characteristics. Linear AR is shown to be a special case of NLAR in [7] (page 28).

\section{APPLICATION OF NLAR TO 2D ROBOT}

As an example of the nonlinear analytical redundancy (NLAR) technique described in the previous section, and 
to demonstrate its potential in robotics, we now derive the NLAR fault detection tests for a two-link, revolute, direct drive manipulator. The robot model was derived for the Integrated Motion Inc. (IMI) robot at Clemson University, as shown in fig. 2.

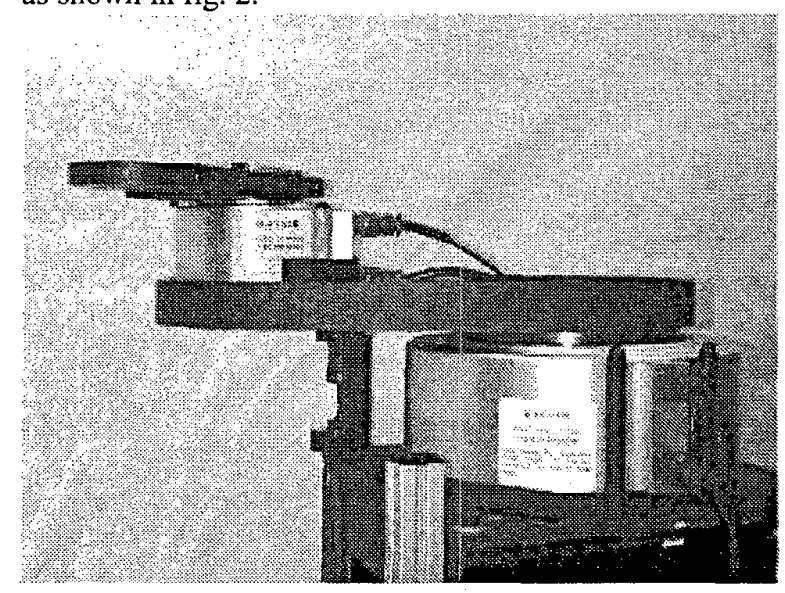

Figure 2: IMI direct-drive manipulator

The model seen in equation (6), from [3] was used to create a simulation of the IMI robot at $1000 \mathrm{~Hz}$. The simulation was used to examine the effect of various simulated faults on the NLAR residuals. Additional redundant sensors were added to show how NLAR deals with sensor redundancy.

$$
\begin{aligned}
& {\left[\begin{array}{l}
u_{1} \\
u_{2}
\end{array}\right]=\left[\begin{array}{cc}
p_{1}+2 p_{3} \cos \left(q_{2}\right) & p_{2}+p_{3} \cos \left(q_{2}\right) \\
p_{2}+p_{3} \cos \left(q_{2}\right) & p_{2}
\end{array}\right]\left[\begin{array}{l}
\ddot{q}_{1} \\
\ddot{q}_{2}
\end{array}\right]} \\
& +\left[\begin{array}{cc}
-p_{3} \sin \left(q_{2}\right) \dot{q}_{2} & -p_{3} \sin \left(q_{2}\right)\left(\dot{q}_{1}+\dot{q}_{2}\right) \\
p_{3} \sin \left(q_{2}\right) \dot{q}_{1} & 0
\end{array}\right]\left[\begin{array}{l}
\dot{q}_{1} \\
\dot{q}_{2}
\end{array}\right] \\
& +\left(\left[\begin{array}{cc}
f_{d 1} & 0 \\
0 & f_{d 2}
\end{array}\right]\left[\begin{array}{l}
\dot{q}_{1} \\
\dot{q}_{2}
\end{array}\right]+\left[\begin{array}{cc}
f_{s 1} & 0 \\
0 & f_{s 2}
\end{array}\right]\left[\begin{array}{l}
\operatorname{sign}\left(\dot{q}_{1}\right) \\
\operatorname{sign}\left(\dot{q}_{2}\right)
\end{array}\right]\right), \\
& \text { where } \\
& p_{1}=3.473 \mathrm{~kg} \cdot \mathrm{m}^{2}, \quad p_{2}=0.193 \mathrm{~kg} \cdot \mathrm{m}^{2}, \\
& p_{3}=0.242 \mathrm{~kg} \cdot \mathrm{m}^{2}, \quad f_{d 1}=1.3 \mathrm{~N} \cdot \mathrm{m} \cdot \mathrm{s}, \\
& f_{d 2}=0.88 \mathrm{~N} \cdot \mathrm{m} \cdot \mathrm{s}, \quad f_{s 1}=1.519 \mathrm{~N} \cdot \mathrm{m}, \\
& f_{s 2}=0.932 \mathrm{~N} \cdot \mathrm{m}
\end{aligned}
$$

The model is in the form of the standard model from equation (3). For NLAR it is reformulated as a statespace control model with the state and output vectors seen in equation (7)

$$
\underline{x}=\left[\begin{array}{llll}
q_{1} & \dot{q}_{1} & q_{2} & \dot{q}_{2}
\end{array}\right]^{T}, \underline{y}=\underline{x} .
$$

The PID controller used only one of the sensors on each joint (the resolver); the redundant tachometers are only used for sensor redundancy in the NLAR tests. Path tracking was through this standard PID error controller run at $100 \mathrm{~Hz}$.

In the test trajectory each joint's input signal was at a different phase and frequency to maximize the variety of joint coupling effects experienced, as shown in eqn (8)

$$
\begin{aligned}
& \theta_{d 1}(t)=0.5 \sin (t / 0.3) \\
& \theta_{d 2}(t)=0.5 \cos (t / 0.4)-0.5 .
\end{aligned}
$$

Data was sampled at $100 \mathrm{~Hz}$, both the controller and the NLAR tests updating every $10 \mathrm{~ms}$. The model was given $300 \mathrm{~ms}$ to settle before testing began.

The NLAR tests themselves were constructed according to the principles outlined in section 2 , using the complete nonlinear control model with the exception of the static friction terms. (The discontinuous nature of static friction invalidates Lie differentiation [4].) The complete derivation of the test residuals are available in [7] (pages 63-73 and Appendix), but space constraints limit what can be presented here. Happily, while the generation of these tests consists of considerable quantities of symbolic mathematical manipulation., all the resulting NLAR tests themselves are scalar equations easily small enough for run time evaluation. Residual complexity is generally proportional to one plus the number of inputs $q$ raised to the power of the number of Lie derivatives taken. However, as some of the more complex tests can be converted to direct sensor comparisons by rank-

\begin{tabular}{|c|}
\hline $\begin{array}{l}\mathrm{R} 1 \text { : Second model equation checked against shoulder } \\
\text { resolver. Tests acceleration of the system. } O(q+1) \text {. }\end{array}$ \\
\hline $\begin{array}{l}\text { R2: Derivative of second model equation checked vs. } \\
\text { shoulder resolver. Tests jerk of the system. } O(q+1)^{2} \text {. }\end{array}$ \\
\hline $\begin{array}{l}\text { R3: Second derivative of second model equation } \\
\text { checked against shoulder resolver. Tests the derivative } \\
\text { of the jerk. } O(q+1)^{3} \text {. }\end{array}$ \\
\hline $\begin{array}{l}\text { R4: Sensor comparison of shoulder tachometer with } \\
\text { derivative of shoulder resolver. } O(q+1) \text {. }\end{array}$ \\
\hline R5: Derivative of R4. $O(q+1)^{2}$. \\
\hline R6: Second derivative of R4. $O$ ( \\
\hline
\end{tabular}
preserving manipulations, this complexity is often reduced. Generally the NLAR tests are about as complex as the system dynamics at the same degree of derivation.

Note also that the number of independent NLAR tests (in this case eleven) depends on the rank of the observability of each sensor. It is interesting to note that the linear version of the system generates fewer tests (eight) due to a lower degree of observability. The following table lists the NLAR tests, their physical interpretations, and their complexity. 
R7: Fourth model equation checked against elbow resolver. Tests the acceleration of the system. $O(q+1)^{2}$.

R8: Derivative of fourth model equation checked against elbow resolver. Tests the jerk of the system. $O(q+1)$.

R9: Sensor comparison of elbow tachometer and derivative of elbow resolver. $O(q+1)$.

R10: Derivative of R9. $O(q+1)^{2}$.

R11: Second derivative of fourth model equation checked against elbow tachometer. Tests the derivative of the jerk. $O(q+1)^{4}$.

Careful reading of the descriptions of the tests will show that they are all model equations, sensor comparisons, or derivatives thereof. It is reassuring that the physical interpretations of the NLAR tests make such intuitive sense. From a mathematical point of view, NLAR takes a complex control model and explicitly separates out tests that are guaranteed to both be independent and span the limits of the information that might indicate deviation from the model. From a practical view, NLAR produces common-sense tests with theoretical support justifying their use. These tests are of the same order of complexity as the system dynamics, allowing for relatively fast evaluation times with respect to the sensor data.

Two examples of these NLAR tests are reproduced below. The first is a test dependent on the system model

$$
\begin{aligned}
& R 1: f_{2}(\underline{x})+u_{1} g_{12}(\underline{x})+u_{2} g_{22}(\underline{x})=\ddot{y}_{1} \\
& =\frac{\left(\begin{array}{l}
\left(p_{3} \sin \left(q_{2}\right)\left(\left(p_{2}+p_{3} \cos \left(q_{2}\right)\right) \dot{q}_{1}+p_{2} \dot{q}_{2}\right)-p_{2} f_{d 1}\right) \dot{q}_{1} \\
+\left(p_{2} p_{3} \sin \left(q_{2}\right)\left(\dot{q}_{1}+\dot{q}_{2}\right)+\left(p_{2}+p_{3} \cos \left(q_{2}\right)\right) f_{d 2}\right) \dot{q}_{2} \\
-p_{2} u_{1}+\left(p_{2}+p_{3} \cos \left(q_{2}\right)\right) u_{2}
\end{array}\right)}{p_{1} p_{2}-p_{2}{ }^{2}-p_{3}{ }^{3} \cos ^{2}\left(q_{2}\right)} .
\end{aligned}
$$

Note that this test is a series of simple operations resulting in a scalar. The second case is a simple sensor comparison

$$
R 4: y_{2}-\dot{y}_{1}=0
$$

\section{ABOUT NLAR RESIDUAL RESULTS}

The observability space does not have a standard set of unit dimensions, and as a result the output of NLAR test residuals is not standardized either. Our example has tests that are in units of velocity, acceleration, jerk, and even the derivative of the jerk. This can be confusing, but for fault detection in practice one is not interested in the units of the test, but only in their relative magnitude. The size of the signal relative to the fault-free 'noise' is of interest, but the units or absolute size are of little consequence.

\section{RESULTS}

The results of our NLAR tests for the two-degree of freedom robot from section three were excellent. Data was collected from the simulated robot every $10 \mathrm{~ms}$ as it tracked a different sinusoidal input with each joint for a period of 10 seconds. NLAR tests were applied and the fault was considered detected if the magnitude of the NLAR result was at least twice the maximum value achieved in a fault free run with the same parameters. More sophisticated detection techniques are certainly possible, but are not developed in this paper.

The following NLAR output is typical of the results:

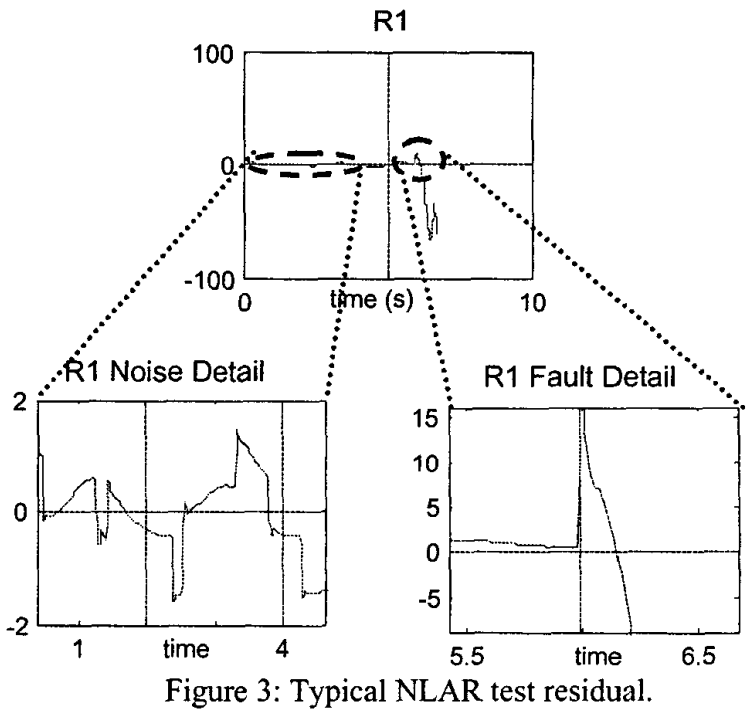

Before the fault occurs at $t=6 \mathrm{~s}$, the NLAR tests mostly show noise-like fluctuations around a mean of 0 . This is to be expected from fault-free NLAR tests as measurement error and unmodeled effects will prevent perfect test residuals in practice. Once the fault occurs this particular test detects it on the same time step. Close examination of the fault detail on fig. 3 shows that the residual at $t=6 \mathrm{~s}$ is about an order of magnitude bigger than the fault free noise.

The table of residuals presented in fig. 4 represents the results of our NLAR tests for a frozen sensor fault - the shoulder resolver is frozen at its current value at $t=6 \mathrm{~s}$. As seen in the following plots, all of the NLAR tests detect the fault very quickly; many of them detect it on the very next time step. This is a promising result, as a frozen sensor does not immediately cause significant tracking errors. 

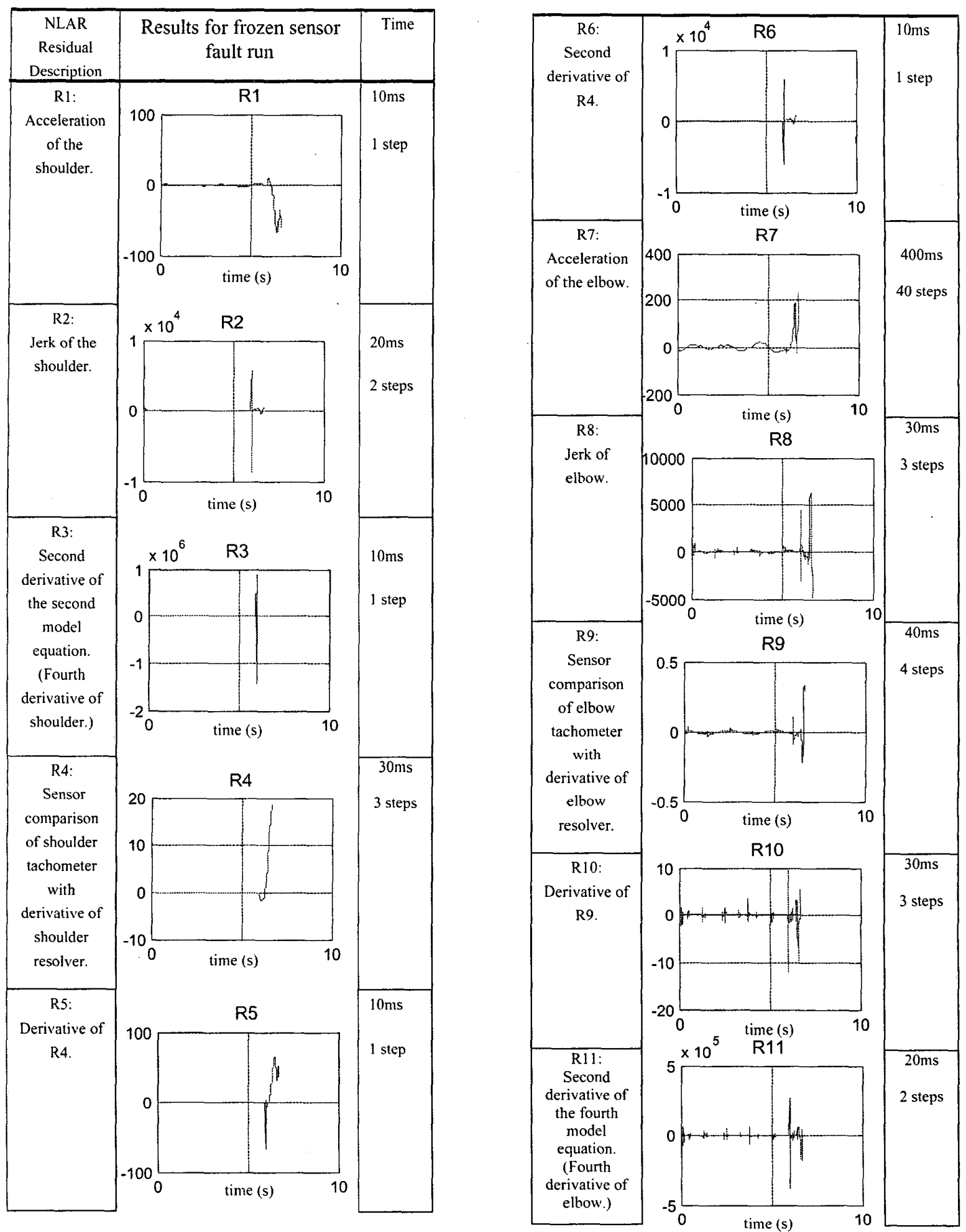

Figure 4: NLAR residuals for a frozen sensor fault. 
The next example, shown in fig. 5, demonstrates a fault that should be very difficult to detect. At $t=6 s$, a sensor drift fault is initiated, and the value for a sensor becomes slowly and smoothly less accurate over time. This kind of fault is much more difficult to detect, since the inconsistencies with respect to the model start at zero and only increase slowly to a detectable value. Since most of the information in the model deals with dynamic behaviors, this slow change produces signals smaller than the noise level on all but one of the NLAR tests. The R1 result in the figure is typical of these. However, R4, which directly tests sensor redundancy, gives a detectable signal. Comparison with the sensor error plot below it on the same figure shows the discrimination of the NLAR test. Before the tracking error is even visible, NLAR has registered a residual two times the fault-free size.

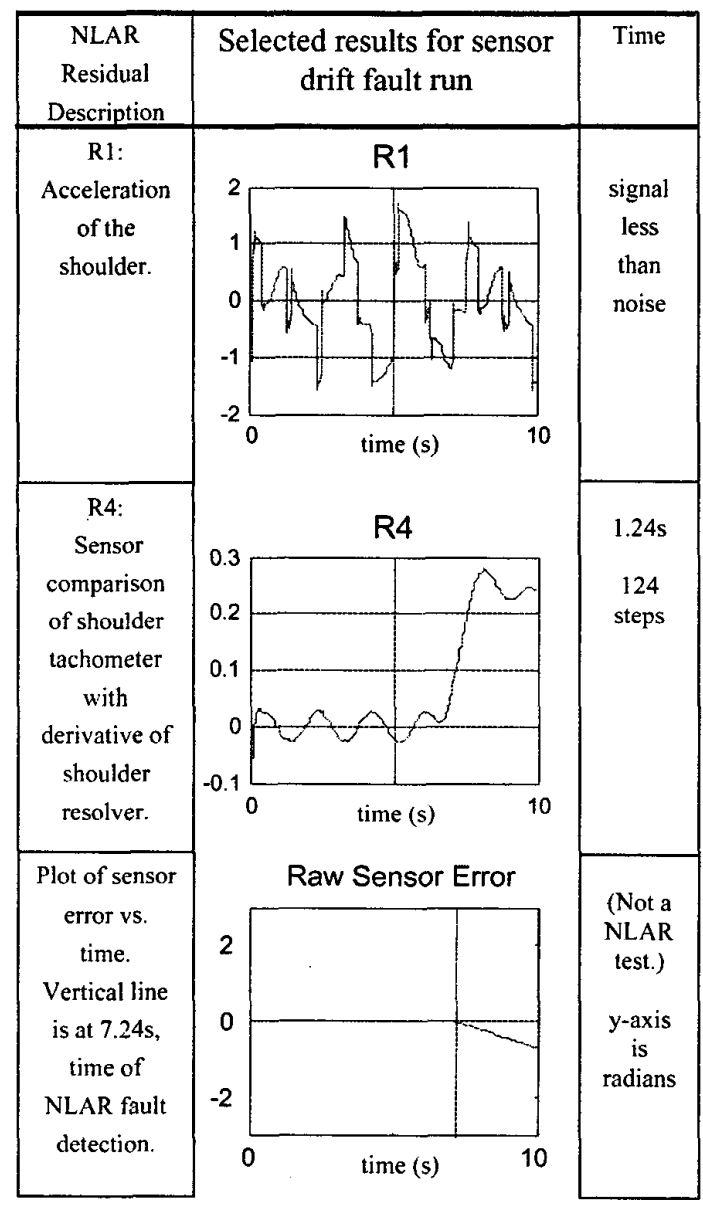

Figure 5: Selected NLAR residuals, sensor drift fault.

The final fault we will examine is a broken motor/drive train fault. In this fault, input to the shoulder motor stops producing torque in the joint at $t=6$ seconds. This fault is interesting in that it produces several NLAR residuals that show signals at the time of the fault that are obvious to the human eye but not twice the magnitude of the fault free case. These faults are labeled as 'detectable' in fig. 6 , as they could be detected by a more sophisticated residual analysis system. However, even assuming the 'detectable' residuals are not exploited, there are several NLAR residuals that detect the fault in a timely fashion using the basic "twice the magnitude" approach.

Figure 7 is a comparison figure showing the improvement in performance of NLAR over linear AR for the same broken motor fault as fig. 6. Note that linear AR produces eight rather than eleven tests, and that four of these are sensor comparisons identical to the NLAR tests and therefore not shown. This leaves four tests that are analogous to NLAR tests but linear in nature. For example, there is a linear AR test that examines the linear model of acceleration of each joint that can be compared to the NLAR test of the nonlinear, model of the same. These analogous tests are compared in fig. 7. It is clear that NLAR outperforms linear AR on all four of these tests. This is likely a result of the poor modeling of the nonlinear coupling and dynamical effects of the manipulator in the linear AR tests.

\section{CONCLUSIONS}

Our new fault detection technique, nonlinear analytical redundancy (NLAR) is a useful technique that provides many powerful theoretical guarantees [1,7]. The ability to see any possible observable deviation from the system model is a very desirable trait in a fault detection system. The ability to deal accurately with nonlinear models is also very desirable both in robotics and many other disciplines $[5,6]$.

The example in this paper is the first application of NLAR to robotics. The residual fault detection results are excellent, showing quick detection of serious faults and sensitivity to even the subtle fault. NLAR also clearly outperforms the linear AR technique. Overall the NLAR technique shows good promise for practical implementation. While the synthesis is nontrivial, involving analysis of the nonlinear dynamics [7] (pages 63-73 and Appendix), the residual tests are quite intuitive and are of the order of complexity of the dynamics or less. These tests are thus comparable in resource usage to a dynamics based controller.

Future work with NLAR is likely to focus on both testing against data from well-modeled nonlinear physical systems and the production of more elaborate systems for analyzing the residual signals. 

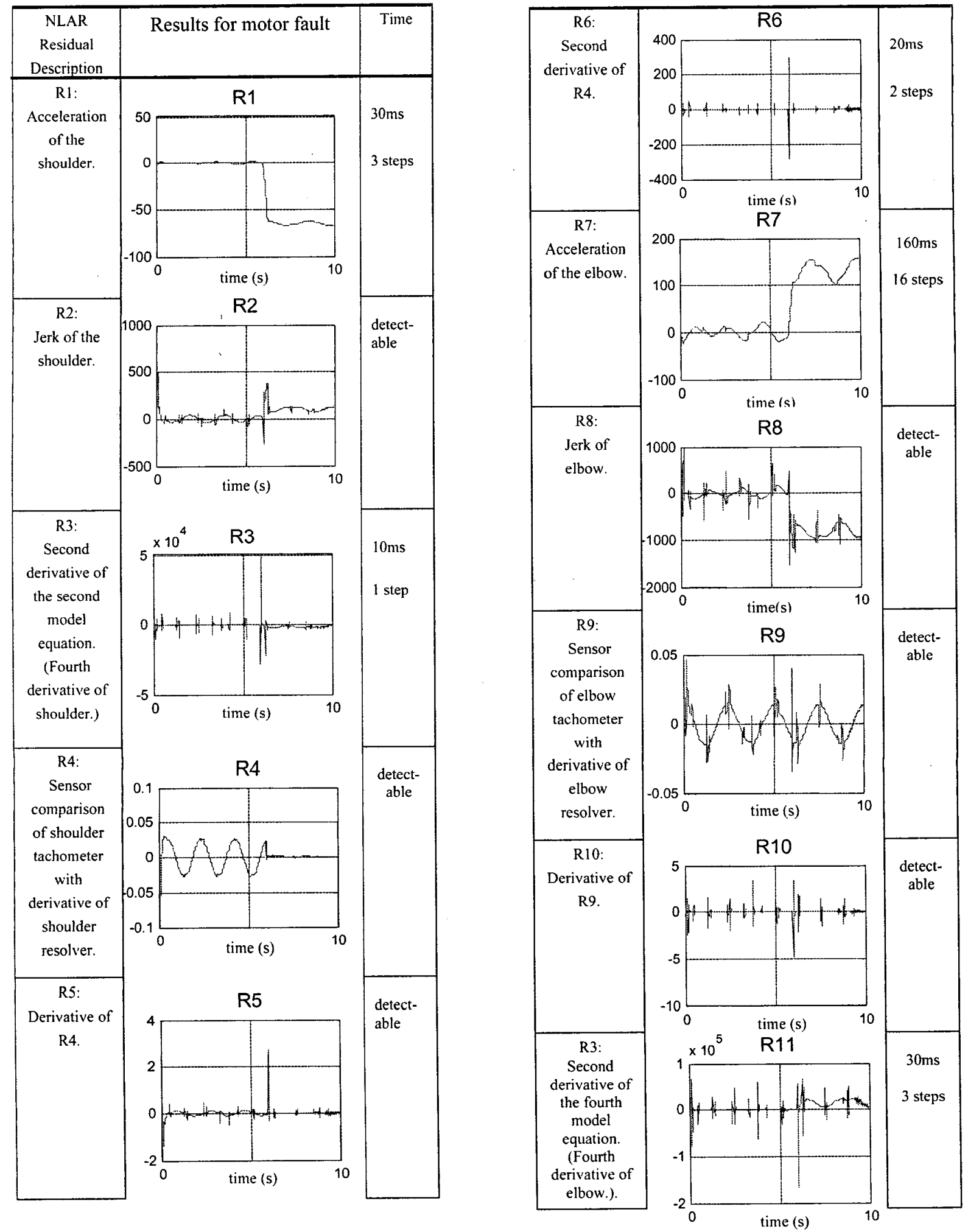

Figure 6: NLAR residuals, motor fault. 


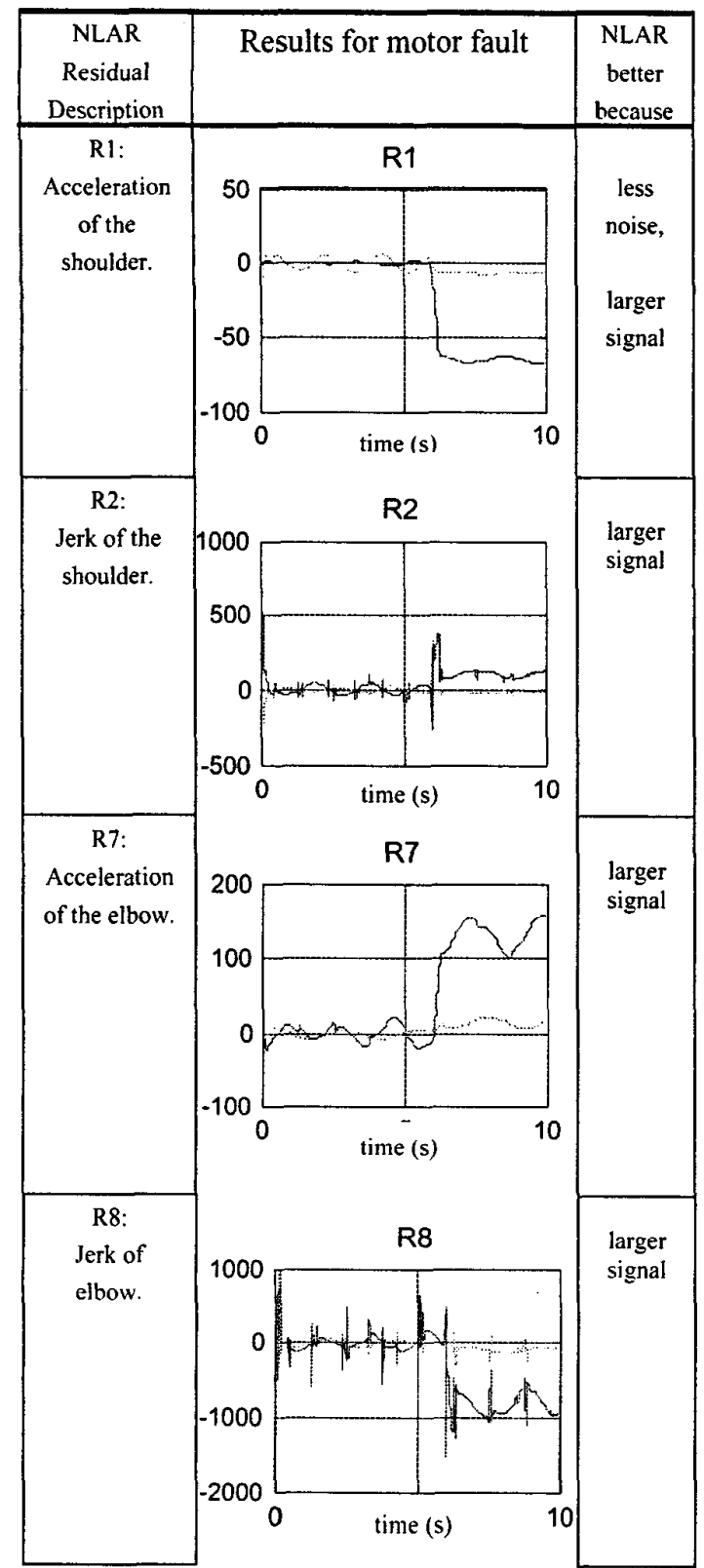

Figure 7: NLAR test result (solid) vs. AR test result (dotted light) for motor fault.

\section{ACKNOWLEDGEMENTS}

This work was supported in part by NASA grant NAG5-9785, NSF/EPSCoR grant EPS-9630167, and DOE contract DE-FG07-97ER 14830.

\section{REFERENCES}

[1] E.Y. Chow and A.S. Willsky. Analytical Redundancy and the Design of Robust Failure Detection Systems. IEEE Transactions on Automatic Control, AC-29(7):603614, July, 1984.

[2] B.S. Dhillon. Robot Reliability and Safety. SpringerVerlag, New York, NY, 1991.

[3] W.E. Dixon, I.D. Walker, Darren M. Dawson, J.P. Hartranft. Fault Detection for Robot Manipulators with Parametric Uncertainty: A Prediction-Error-Based Approach. IEEE Transactions on Robotics and Automation, 16(6):689-699, 2001.

[4] A. Isidori. Nonlinear Control Systems. SpringerVerlag, London, UK, 1995.

[5] M.L. Leuschen, I.D. Walker, J.R. Cavallaro, R. W. Gamache, M. Martin. Experimental AR Fault Detection Methods for a Hydraulic Robot. In Proceedings of the 18th International Systems Safety Conference, pp. 402409, Fort Worth, TX, Sept 2000.

[6] M.L. Leuschen, I.D. Walker, J.R. Cavallaro. Nonlinear Analytical Redundancy for Fault Detection. (Submitted to) IEEE Transactions on Automatic Control, 2002.

[7] M. L. Leuschen. Derivation and Application of Nonlinear Analytical Redundancy. Doctoral thesis, Rice University, ECE Dept, Houston, TX, 2001.

[8] M. Staroswiecki, G. Comtet-Varga. Analytical Redundancy Relations for Fault Detection and Isolation in Algebraic Dynamic Systems. Automatica, v37, n5, pp 687-699, 2001.

[9] M.L. Visinsky, J.R. Cavallaro, and I.D. Walker. Robotic Fault Detection and Fault Tolerance: A Survey. Reliability Engineering and System Safety, 46(2):139-158, 1994.

[10] A.N. Zhirabok, O.V. Preobragenskaya; Instrument Fault Detection in Nonlinear Dynamic Systems. Proceedings of the IEEE International Conference on Systems, Man, and Cybernetics. Part 5 (of 5), pp 114 119. 1993. 